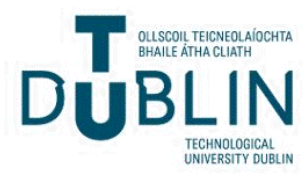

Technological University Dublin

ARROW@TU Dublin

2014-12-31

\section{Contactless Measurement of Angular Velocity using Circularly Polarized Antennas}

\author{
Vit Sipal \\ Technological University Dublin, vit.sipal@tudublin.ie \\ Adam Narbudowicz \\ Technological University Dublin \\ Max Ammann \\ Technological University Dublin, max.ammann@tudublin.ie
}

Follow this and additional works at: https://arrow.tudublin.ie/engscheleart2

Part of the Electrical and Electronics Commons, and the Electromagnetics and Photonics Commons

\section{Recommended Citation}

Sipal, V.; Narbudowicz, A.Z. \& Ammann, M.J., (2015) Contactless Measurement of Angular Velocity Using Circularly Polarized Antennas, Sensors Journal, IEEE , vol.15, no.6, pp.3459,3466, doi:10.1109/

JSEN.2014.2385815

This Article is brought to you for free and open access by the School of Electrical and Electronic Engineering at ARROW@TU Dublin. It has been accepted for inclusion in Articles by an authorized administrator of ARROW@TU Dublin. For more information, please contact arrow.admin@tudublin.ie, aisling.coyne@tudublin.ie, gerard.connolly@tudublin.ie.

Funder: Irish Research Council, Science Foundation Ireland 


\title{
Contactless Measurement of Angular Velocity using Circularly Polarized Antennas
}

\author{
V. Sipal, Member IEEE, A. Narbudowicz, Member IEEE, and Max. J. Ammann, Senior Member IEEE
}

\begin{abstract}
An innovative method to measure angular velocity using circularly-polarized antennas is proposed. Due to the properties of circular polarization, the angular velocity is frequency modulated (FM) on a wireless carrier. This enables a low-cost precise continuous measurement of angular velocity using a standard FM demodulator. The hardware can be easily adapted for both high and low angular velocity values. The precise alignment angle between the antennas can be determined if the initial antenna orientation is known. The angular error is shown to be less than $1.5^{\circ}$ for antennas with a $1 \mathrm{~dB}$ axial-ratio. The basic method is applicable for a fixed distance between the antennas. Therefore, an advanced method which provides data for variable distances is also presented.
\end{abstract}

Index Terms - angular velocity, measurement technique, antennas, rotation sensor

\section{INTRODUCTION}

$\mathrm{T}$ HE measurement of angular velocity is a well-studied topic because tachometers are required in a plethora of applications across many industries. Most devices rely on counting pulses which are associated with a displacement by a fixed angle [1]-[3]. The source of these pulses can be a magnetic field or a reflected optical signal. The main drawback of this method is the inability to provide continuous information about the angle between two consecutive pulses. As a result, the measurement of small changes in angular velocity or angular displacement requires a greater number of pulse sources. For high precision systems, this becomes a challenge [1],[2].

This limitation is addressed in this paper, which introduces a novel sensor concept for the continuous measurement of angular displacement using electromagnetic fields in the $\mathrm{GHz}$ region. Using an electromagnetic field in the $\mathrm{GHz}$ region was presented recently in [4]-[6], where the rotation changes the transfer function of a circuit based on transmission lines.

In contrast, the sensor introduced here works on a fundamentally different principle. It is contactless and it relies on the establishment of a radio link between two circularly

Manuscript received June 30, 2014. The work was funded by the Government of Ireland Fellowship in Engineering, Science and Technology funded by the Irish Research Council and by Science Foundation Ireland under Grants No. 10/CE/I1853 and and No. 13/TIDA/I2746.

V. Sipal, A. Narbudowicz and M. J Ammann are with the Dublin Institute of Technology, Dublin 8, Ireland (phone: +3531 402 4905; fax: +3531402 4690; e-mail: vit.sipal@ dit.ie). polarized (CP) antennas. If one of these antennas is rotated, the link transfer function is changed and this effect is proposed for the measurement of angular velocity.

The proposed sensor is unique, as it offers the flexibility to measure angular velocities ranging from very low speeds up to $1,000,00 \mathrm{rpm}$. The hardware modifications associated with different velocity ranges are minor.

The proposed system allows constant monitoring of the angle as a function of time and it is possible to get precise information about the angle and angular velocity regardless of the distance between the transmit and receive pars of the sensor. Moreover, it can be integrated with existing wireless links (e.g. WiFi or Bluetooth) used to send data from other sensors. Finally, a single receiver can measure the rotation velocity of multiple transmitters, significantly saving costs.

To the best of our knowledge, sensors offering comparable capabilities have not been reported in the open literature.

First the underlying physical principle is introduced with an example outcome and the system design is presented in Section II. The accuracy is discussed in Section III and Section IV shows how to implement the sensor for a varying distance between the antennas.

\section{SENSOR CONCEPT}

\section{A. Physical Principle}

To demonstrate the principles of operation of the proposed sensor, a system description of a communication link between two antennas is investigated. A general link with two arbitrary antennas is shown in Fig. 1. The transfer function $H(f)=$ $S_{\text {out }}(f) / S_{\text {in }}(f)$ between the antennas can be described as [7]:

$$
H(f)=\frac{c_{0} \exp \left(-j 2 \pi \frac{f}{c_{0}}\right)}{j 4 \pi f r}\left(\begin{array}{c}
H_{2 X}(f) \\
H_{2 Y}(f)
\end{array}\right)^{T}\left(\begin{array}{cc}
\cos \varphi & \sin \varphi \\
\sin \varphi & -\cos \varphi
\end{array}\right)\left(\begin{array}{c}
H_{1 X}(f) \\
H_{1 Y}(f)
\end{array}\right)
$$

where $f$ is the frequency, $c_{0}$ is the speed of light, $r$ is the distance between antennas, and $H_{i X}$ is the transfer function between the input voltage wave at the antenna port $i$ and the electric field $E_{i X}$ in $x$-direction (in antenna's respective coordinates) caused by the input voltage wave [8]. $H_{i Y}$ is the equivalent quantity for the second polarization orientation in $y$-direction [7], [8]. The middle term accounts for the mutual position of the coordinates of the antennas considering the rotation of one coordinate system around the $z_{\mathrm{i}}$ axis by angle $\varphi$ (see Fig. 1).

Eq. (1) describes a generic link between two antennas. For a special case of using circularly-polarized antennas, it can be simplified as discussed next. 

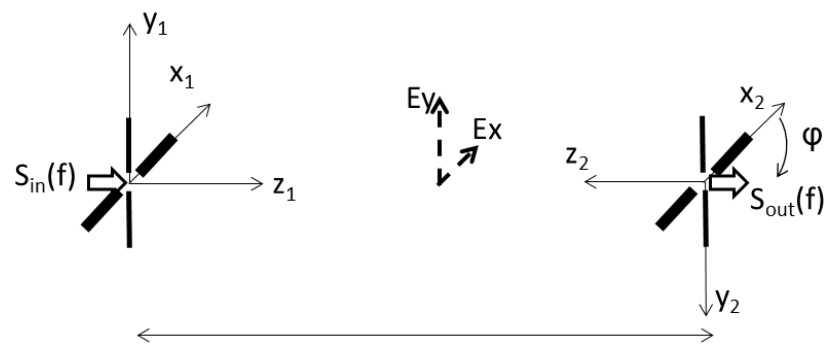

Fig. 1. System overview for a transmission between two antennas. Different lines for the antennas highlight that for now, there is no constraint on antenna properties in either polarization direction.

A circularly-polarized electromagnetic wave exhibits special properties because the vector of the electric field scribes a circle on the wave-front perpendicular to the direction of the propagation ( $x y$ planes in Fig. 1) [9]. Circularly-polarized antennas are found in many applications such as in satellite communications or in some modern wireless systems [9], due to the rotating electric field.

In terms of the antenna transfer functions in (1), circularpolarization means that there is a fixed relation between the electric field components the in $x$ - and $y$-directions: $H_{\mathrm{iX}}(f)=$ $\pm j \operatorname{sgn}(f) H_{\mathrm{iY}}(f)$, where \pm determines the direction of rotation (i.e. right- or left-hand rotation) [7]. For the sake of simplicity, the rest of the paper considers only positive frequencies, i.e. the sign function $\operatorname{sgn}(f)$ will not be considered. Then, (1) is transformed into [7]:

$$
H(f)=\frac{c \exp \left(-j 2 \pi \frac{f}{c} r\right)}{\mathrm{j} 2 \pi f r} H_{2 X}(f) H_{1 X}(f) \exp ( \pm j \varphi)
$$

The first physical meaning of (2) is the fact that the amplitude of the received signal is independent of the rotation of the antennas in the $x y$ plane. This is used e.g. in GPS localization links. The second physical meaning relates to the phase term in (2). It is apparent, that for constant $r$ the phase is directly proportional to the physical angle $\varphi$ between the two antennas. To our best knowledge, this property is known but only considered for its negative effects such as signal distortion [10] or reduced precision of GPS systems [11].

The exception is our work [7], which shows that (2) holds also for near-field coupled antennas and can therefore be used for the design of novel phase shifters. In contrast, here we use (2) directly for antennas in the far-field and aim to use the phase dependency for sensor applications.

It is also noted that the rotation of the circularly-polarized field is exploited in some antenna arrays where rotation of the elements is used to suppress mutual coupling or to introduce a phase shift for beam-steering [9].

\section{B. System architecture}

The practical importance of (2) is when the phase is observed because in the case of constant range $r$ between the transmitter and the receiver, the phase of the transfer function $\measuredangle H(f, t)$ at time $t$ is given as:

$$
\measuredangle H(f, t)=\measuredangle H(f, t=0)+\varphi(t)
$$

where $\varphi(t)$ describes the angle of physical rotation between the two antennas at any given time $t ; \measuredangle H(f, t=0)$ is the phase of the transfer function at the beginning of the

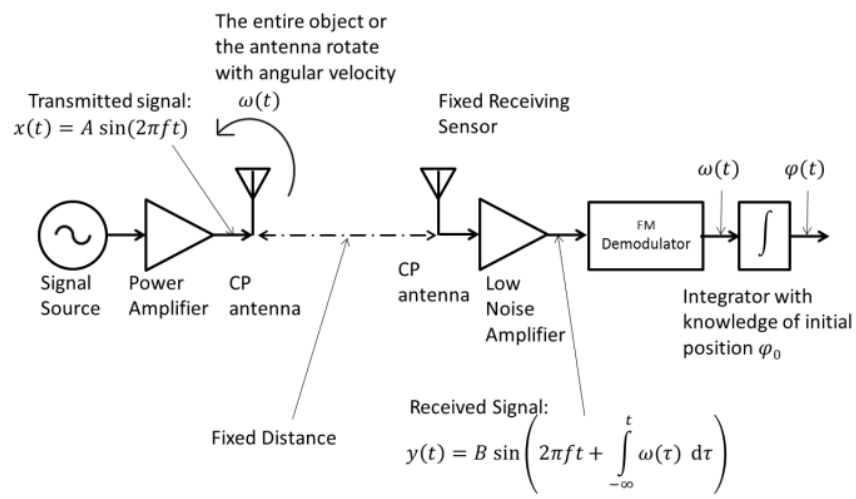

Fig. 2. Example system architecture for angular velocity measurement.

observation given as

$\measuredangle H(f, t=0)=\measuredangle\left[\frac{c \exp \left(-j 2 \pi \frac{f}{c} r\right)}{\mathrm{j} 2 \pi f r} H_{2 X}(f) H_{1 X}(f)\right]+\varphi(0)$

For the sake of brevity, let us define a system parameter $\theta_{S Y S}$ :

$\theta_{S Y S}=\measuredangle\left[\frac{c \exp \left(-j 2 \pi \frac{f}{c} r\right)}{\mathrm{j} 2 \pi f r} H_{2 X}(f) H_{1 X}(f)\right]$

From (3) it is apparent that observation of the phase of the transfer function between two circularly-polarized antennas provides information about the physical angle between two antennas. When the angle is known, calculation of the angular velocity $\omega(t)$ is straightforward.

An example of a system capable of measuring the angle and angular velocity is shown in Fig. 2. It consists of a transmitter placed on the rotatable object and a fixed receiver. The rotatable object and the antenna therefore rotate with the same angular velocity

The transmitter is likely to be of low complexity comprising only a circularly polarized antenna, a signal source and the necessary amplification circuitry. As shown in Fig. 2, the signal source generates a simple harmonic signal $x(t)=$ $A \sin (2 \pi f t)$, which is fed to the antenna. In practical implementations, more complex signals are envisaged.

The transmitter is placed on a rotating object ideally so that the axis of the rotation (dash-dot line in Fig. 2) is in line with the antenna main radiation direction (chosen as the best combination of good gain and low axial ratio).

In the simple case presented above, the transmitter can be represented by compact circularly-polarized antennas (e.g. [12],[13]) and transmitter circuitry which can be integrated as a flip chip into the antenna structure.

The antennas radiate in a direction aligned with the axis of rotation. Considering (2)-(4) the received signal at the port of the receiving antenna is given by:

$$
y(t)=B \sin (2 \pi f t+\measuredangle H(f, t=0)+\varphi(t))
$$

which can be rewritten as:

$$
\begin{aligned}
y(t) & =B \sin \left\{2 \pi f t+\theta_{S Y S}+\varphi(0)+\varphi(t)\right\}= \\
& =B \sin \left\{2 \pi f t+\theta_{S Y S}+\int_{-\infty}^{t} \omega(\tau) \mathrm{d} \tau\right\}
\end{aligned}
$$

In (7) it is observed that the angular velocity $\omega(t)$ is frequency modulated on the received signal. As a result, frequency demodulation of the received signal provides information about the angular velocity. The frequency demodulation can easily compensate for $\theta_{S Y S}$ (in the same way 
as standard FM radio receivers receive the same information regardless their distance from the transmitter). The previous statement is valid as long as the range $r$ between the transmitter and receiver does not change, i.e. as long as $\theta_{S Y S}$ remains constant. It is noted that the case of variable $\theta_{S Y S}$ due to a change in the range is further discussed in section IV.

When the angular velocity $\omega(t)$ is known, the angle $\varphi(t)$ can be determined in a straightforward manner when the initial system condition $\varphi(0)$ is known. Therefore, the same method applies to the contactless measurement of the angle between two objects as well as the measurement of angular velocity.

\section{Proof of concept}

The system described in section II.B operates only under the assumption that equations (1)-(4) are valid not only theoretically, but also hold with sufficient precision for practical wireless links between two antennas. This is expanded in Section III.

To test the concept, the phase of the transfer function for a link using two circularly-polarized antennas facing each other is investigated. One antenna is fixed whereas the second is positioned on a rotary platform. The platform allows rotation over a number of angles $\varphi$ with a $0.5^{\circ}$ accuracy. The phase is measured using a Rohde \& Schwarz ZVA 40 Vector Network Analyzer (VNA) and is compared to the ideal case. All interfaces use standard SMA connectors, which provide mechanical stability and good connectivity at higher frequencies. To allow unobstructed movement, the rotating antenna is connected to the VNA via a rotary joint.

The rotary joint was used for the proof-of-concept measurements, in order to allow connection between the rotating antenna and the stationary vector network analyzer. The rotary joint provides a coaxial connection between a fixed part of the set-up and a rotating part. The use of such joints is
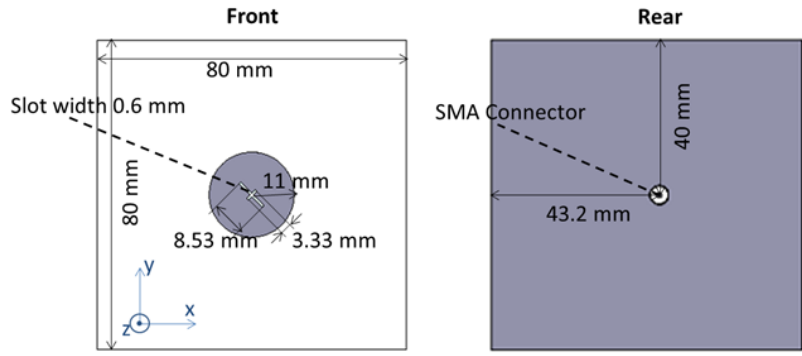

Fig. 3. Example antenna providing right hand circular-polarization

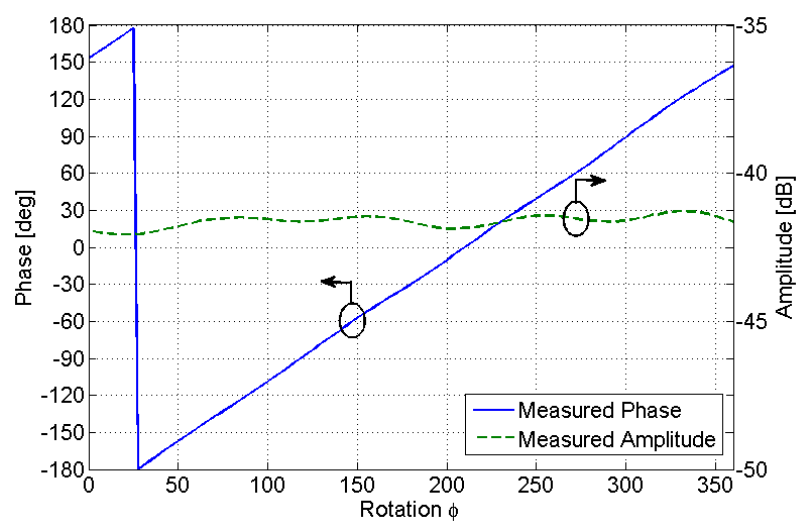

Fig. 4 Transmission between two antennas from Fig. 3 spaced $75 \mathrm{~cm}$ apart The transfer function is measured at $3.575 \mathrm{GHz}$. common in antenna measurements where they allow repeatable automated antenna revolution around a rotary axis.

Since the platform moves between discrete angles $\varphi$, the time $t$ can be removed from (3) resulting in the following linear relationship:

$$
\measuredangle H(f, \varphi)=\measuredangle H(f, \varphi=0)+\varphi
$$

Eq. (8) means that in order to prove that concept from Section II.B is valid, the phase of the transfer function between two circularly-polarized antennas should change linearly as one of the antennas is rotated by angle $\varphi$.

The experiment for validation of (8) is introduced in Figs. 3 and 4. Fig. 3 shows a circular microstrip patch antenna providing Right-Hand Circular Polarization (RHCP) which was fabricated on FR-4 substrate of thickness $1.57 \mathrm{~mm}$.

Fig. 4 then presents the phase measured by the VNA at $3.575 \mathrm{GHz}$. For the measurement two antennas were placed 75 $\mathrm{cm}$ apart (facing each other) in an anechoic chamber. One antenna was rotated by $360^{\circ}$ in 145 discrete steps while the phase of the transfer function, $\measuredangle H(f, \varphi)$ was recorded. Fig. 4 shows the recorded phase as a function of rotation angle $\varphi$. The plot clearly displays linear phase change with rotation around the axis connecting both antennas, i.e. confirming that the underlying theoretical assumption can be achieved for a practical scenario.

It is noted that the phase value $\measuredangle H(f, \varphi=0)$ for position angle $\varphi=0^{\circ}$ is effectively $\theta_{S Y S}$ from (5). In other words it is determined by the antenna transfer functions and by the range, as a result it is a random variable with uniform distribution, but constant for a fixed distance system. Most importantly, the change in rotation angle $\Delta \varphi$ equals the same change in phase of the transfer function $(\measuredangle H(f, \varphi))$.

The plot also confirms that the amplitude of the received signal is constant with amplitude variance $\leq 0.75 \mathrm{~dB}$ for full rotation.

\section{Benefits of the system}

The first and main benefit of this technique is the ability to process high angular speeds. For instance rotation at $1,000,000 \mathrm{rpm}$ corresponds to frequency modulation by a $16.67 \mathrm{kHz}$ signal. This is easily processed by modern wireless systems which cope with bandwidths $>500 \mathrm{MHz}$ [14]. At the same time, precision phase-locked-loops can be used to follow slow rotations. In other words, the receiver can be adapted to the application at a reasonably low cost.

For larger angular velocities, the mounting of the transmitting section on the rotating object, e.g. a turbine, can become more challenging. However, one should bear in mind that the antenna is placed on the axis of the rotation, hence the centripetal forces will not be large as long as electrically small and lightweight antennas are employed.

The second benefit is the continuous observation of $\omega$ as a function of time. As the frequency modulation is continuous, the temporal resolution of changes in $\omega$ depends only on the system bandwidth and the speed of analogue-to-digital conversion. Considering that ultra wideband radio systems can easily achieve a sampling of 1 GSps [14], it is suggested that the currently available sampling rate is sufficient for precise 
observation of the rotation to detect deviations from ideal rotation with constant $\omega$, even during a single rotation.

The third benefit is the ability to integrate the system with an existing wireless link, i.e. the transmitter can send a modulated signal with data e.g. about the temperature. This modulation is overlaid by a second modulation due to the rotation. At the receiver, both modulations are separable, thus providing more information from the existing wireless link.

Finally, it can operate over longer ranges and that antenna alignment is less critical than for pulse counters using lasers.

So far, the sensor assumed ideal conditions: perfect circularly-polarized antennas and a fixed distance between the transmitter and receiver. The impact of the non-ideal scenarios is discussed in Sections III and IV.

\section{SENSOR PRECISION}

\section{A. Impact of antenna properties}

The physical principle introduced in Section II.A considers the use of ideal circularly-polarized antennas. However, practical antennas typically deviate from this. The relationship between the transfer function for the $x$ and $y$ polarizations (notation according to Fig. 1 and (1)) can be described as:

$$
H_{\mathrm{iX}}(f)=j A_{i} H_{\mathrm{iY}}(f)
$$

where $A_{i}$ is a complex number describing the amplitude and phase deviation from an ideal circularly-polarized antenna.

For the rest of the paper, we will assume that $A_{\mathrm{i}}$ is a real number. For $\left|A_{\mathrm{i}}\right|>1,\left|A_{\mathrm{i}}\right|$ corresponds to the axial-ratio of the antenna, which is a parameter describing its polarization purity [9]. For $\left|A_{\mathrm{i}}\right|<1$, the antenna's axial-ratio is $1 /\left|A_{\mathrm{i}}\right|$. For $A_{\mathrm{i}}=0$, the antenna exhibits linear polarization, i.e. the excited field has only one component.

This does not mean the loss of generality since for complex $A_{\mathrm{i}}$, it is always possible to apply a coordinate transformation (antenna rotation) so that the transformed $A_{i}^{\prime}$ is real.

Substituting (4) into (1) and using $H$ as an abbreviation for $H(f)$ changes (2) into:

$H=\frac{c \exp \left(-j 2 \pi \frac{f}{c} r\right)}{\mathrm{j} 2 \pi f r} H_{2 X} H_{1 X}\left[\left(1+A_{1} A_{2}\right) \cos \varphi+j\left(A_{1}+A_{2}\right) \sin \varphi\right]$

There are three cases for this result (5):

1) $A_{1}=A_{2}=0$, i.e. both antennas are linearly-polarized. In this case the phase of the transfer function $\measuredangle H(f)$ is independent of the angle $\varphi$ which only impacts on the amplitude. This is not suitable for the proposed method.

2) $A_{1}= \pm 1, A_{2}=\mp 1$, i.e. both antennas are ideally circularly-polarized but in the opposite sense. In such a case, $H(f)=0$ and communication cannot be established.

3) For all other cases, Eq. (5) describes an ellipse in the complex plane with major and minor axes given by as $\left|1+A_{1} A_{2}\right|$ and $\left|A_{1}+A_{2}\right|$.

The last case is therefore relevant for the use as a sensor. In practice it makes little sense to consider a link between two antennas with opposite polarization sense $\left(A_{1} A_{2}<0\right)$, as the amplitude of $H(f)$ is low. Henceforth, the paper will focus on subcases $A_{1} A_{2}>0$, or $A_{1} A_{2}=0$ with $A_{1} \neq A_{2}$, i.e. both antennas have the same sense of the polarization, or only one antenna is circularly-polarized and the second one is linearly polarized.

For these cases, the phase of the transfer function $\measuredangle H(f, \varphi)$ is a function of the rotation $\varphi$ but not necessarily a linear function. Eq. (5) describes and ellipse. When the antenna is rotated by angle $\Delta \varphi$, the value of $H$ moves along the ellipse.

It is apparent that if at least one antenna is a perfect circularly-polarized antenna, i.e. $\left|A_{\mathrm{i}}\right|=1$, the ellipse is a circle and the relationship between $\measuredangle H(f, \varphi)$ and $\varphi$ is a linear function as described in (3) for the right-hand circularlypolarized case. For non-perfect antennas the function will deviate from the ideal linear relationship. Sections III.B and III.C quantify this deviation for practical axial-ratio values.

\section{B. Link between two CP antennas}

For this case, the position on the ellipse remains a function of rotation angle $\varphi$, but (3) is only an approximation of the result. The error of this approximation depends on the axial ratio. This is further studied in the following experiment.

For two antennas spaced by $75 \mathrm{~cm}$ (as seen in Fig. 3), the transfer function was recorded for 301 frequency points between $3.45 \mathrm{GHz}$ and $3.75 \mathrm{GHz}$ for a full rotation stepped by $2.5^{\circ}$. For each frequency the phase of the transfer function, $\Varangle H(f, \varphi)$, was recoded as a function of angle $\varphi$ (same as in Fig. 3). An example of a practical deviation for imperfect axial-ratios is shown in Fig. 5. It shows the same information as Fig. 3 but for a frequency $3.51 \mathrm{GHz}$, where the antennas have a poor axial-ratio of $8 \mathrm{~dB}\left(\left|A_{i}\right|=2.5\right)$, and compares it with the ideal phase dependency from (3). For the ideal dependency in Fig. 5, Ł $H(f, \varphi=0)$ was estimated from the measured results using the Minimum Mean Square Error (MMSE) curve fitting tools in Matlab.

The difference between the ideal function from (8) and the measured phase was quantified using the mean absolute error $e$, which is defined as follows:

$$
e=\frac{1}{N} \sum_{i=1}^{N}\left|\varphi_{M}(i)-\varphi_{I}(i)\right|
$$

where $\varphi_{M}$ is the measured phase, $\varphi_{I}$ is the ideal phase for $i$ th angular step. $N$, number of steps for a full rotation, is 145 .

Fig. 6 presents the measured mean absolute error (5) for transmission between two antennas spaced by $75 \mathrm{~cm}$ for the measured frequency range $3.45 \mathrm{GHz}-3.75 \mathrm{GHz}$. This plot also relates the mean absolute error with the measured axial ratio of the antennas. Fig. 6 shows the strong link between the

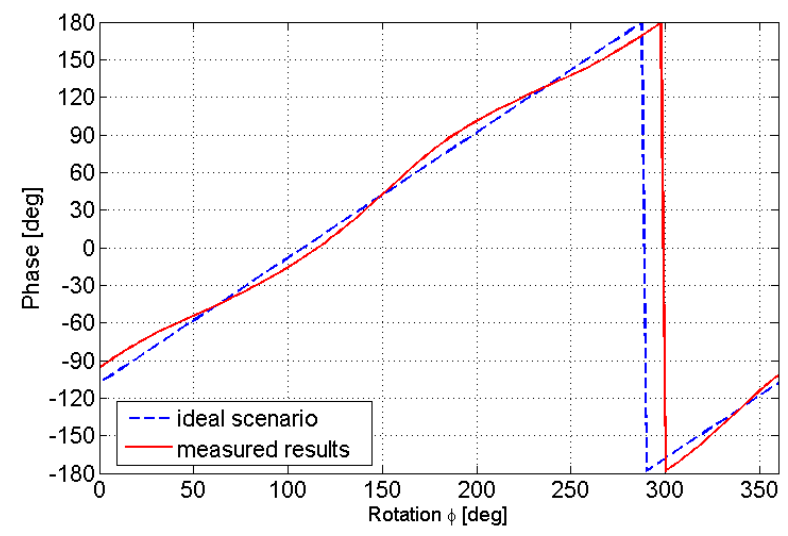

Fig. 5. Example of real phase relationship compared to the ideal scenario. 


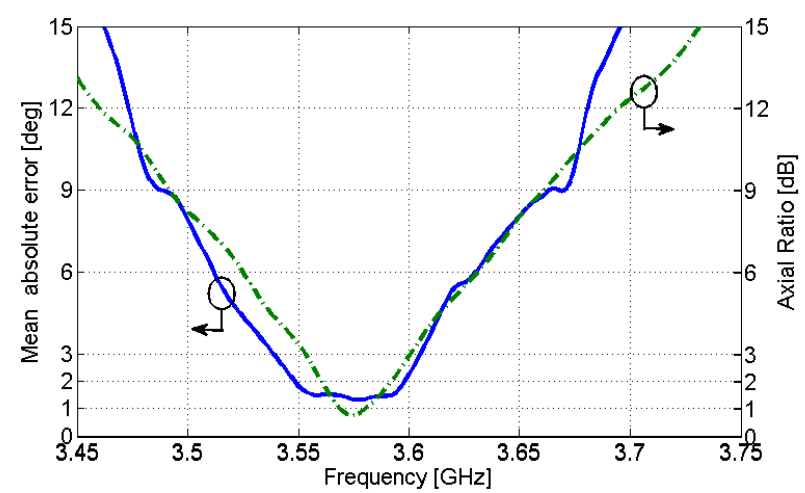

Fig. 6. Mean absolute error for a link between two CP antennas.

angular precision and the axial-ratio. Moreover, it shows that mean absolute error of the phase is less than $2^{\circ}$ for the case when both antennas have an axial ratio less than $3 \mathrm{~dB}$, which is a common requirement for circularly-polarized antennas.

It is crucial to note that the error presented in Fig. 6 is only in part due to the antennas and it is influenced by other sources of uncertainty such as the precision of the rotary platform (our platform had a guaranteed precision of less than 0.5 degrees), the imperfect phase response of the rotary joint used to enable the rotation of the antenna and the phase noise of the VNA. These uncertainties explain the flattening of the mean absolute error at value of approx. $1.5^{\circ}$. The existence of these uncertainties especially of the one due to the rotary joint is proven in Fig. 7.

An ideal rotary joint should have a constant transfer function regardless its rotation orientation, i.e. the phase and the amplitude should not be impacted by the rotation. The measured phase error of the rotary joints used (along with and phase-noise of the measurement) is presented in Fig. 7.

Fig. 7 presents the mean absolute error for a measurement where the link between two antennas was replaced by a coaxial cable between two rotary joints. The setup is shown in the inset of Fig. 7.

Whilst in the wireless link, one rotary joint is sufficient because of the free-space between the antennas. In the measurements from Fig. 7, two rotary joints are necessary to allow for a free rotation of the coaxial cable between two fixed ports of the network analyzer without twisting any of the cable segments used.

During the measurement, the middle segment of the coaxial cable was connected to the rotary platform and rotated by a full $360^{\circ}$. The measured mean absolute error in the frequency range of interest is approx. $0.75 \mathrm{deg}$.

It is noted that, Fig. 7 does not directly quantify the measurement error shown in Fig. 6. Firstly, the measurement in Fig. 7 is not impacted by the positioning error of the rotary platform. Secondly, it uses two rotary joints (both rotating) whereas for measurement from Fig. 6 only one rotary joint was used because the link between the antenna was wireless. However, Fig. 7 confirms the presence of imperfections in the rotary joints. Hence it explains the flattening of the error in Fig. 6. It should be noted, that the rotary joint was necessary for proof of concept and would not be used in practical

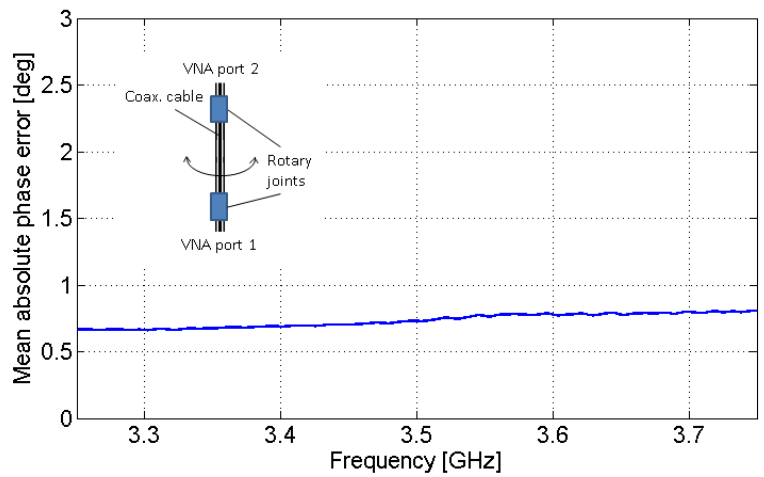

Fig. 7. Mean absolute error caused by imperfections of two rotary joints and the phase noise of the VNA. Inset shows the measurement setup.

contactless implementations, which should further improve resolution.

\section{Link between an LP and a CP antenna}

The special case when only one antenna is circularlypolarized is described. This case is interesting because of the simplicity of one of the antennas, i.e. its potential for low cost sensors.

In such a case one of the axial-ratios in (5) is equal to infinity. For instance for $A_{1}=0$, Eq. (5) becomes:

$$
H=\frac{c \exp \left(-j 2 \pi \frac{f}{c} r\right)}{\mathrm{j} 2 \pi f r} H_{2 X} H_{1 X}\left[\cos \varphi+j A_{2} \sin \varphi\right]
$$

Eq. (6) is still an equation of an ellipse but it can be observed that it is significantly more susceptible to change in axial-ratio of the circularly-polarized antenna, i.e. the axis ratio of the ellipse is $\left|A_{2}\right|$ instead of $\left|A_{1}+A_{2}\right| /\left|1+A_{1} A_{2}\right|$ as for the case of two circularly-polarized antennas. For instance for circularly-polarized antennas with axial-ratio of $3 \mathrm{~dB}\left(A_{i}=\right.$ $\sqrt{2}$ ), the axis ratio of the ellipse in the phase domain is $\sqrt{2}$ for a link between linearly-polarized and circularly-polarized antennas whereas it is only 1.06 for a link between two circularly-polarized antennas. This means that the ellipse in the phase domain is expected to be more distorted for a link with one linearly-polarized antenna. As a result the phase angle relation used by this technique will be less linear.

This is shown in Fig. 8 which presents the same experiment as in Fig. 6 but for a link between a circularly-polarized and a linearly-polarized antenna. The linearly-polarized antenna had the same dimensions as the antenna in Fig. 2 but fabricated

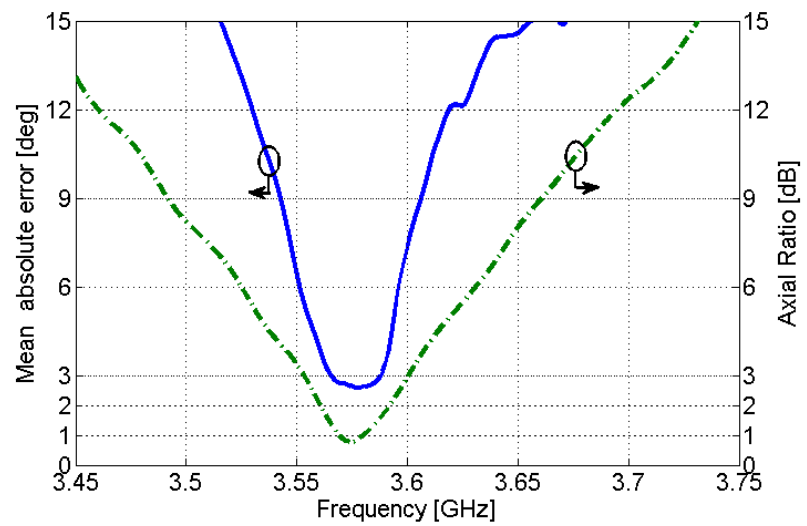

Fig. 8. Mean absolute error for a link between a LP and a CP antenna. 
without the slot in the circular patch. It is apparent that the mean absolute error is significantly worse than when using two circularly-polarized antennas.

\section{System implications}

This section discussed the impact of axial-ratio on the method angular precision. The results show that the phase error depends on the antenna axial-ratios and it is shown that from the system perspective it is beneficial to employ two circularly-polarized antennas for the wireless link. This setup reduces the error compared to a link between a linearlypolarized and a circularly-polarized antenna.

It is shown that for a link between two circularly-polarized antennas meeting the standard $3-\mathrm{dB}$ axial ratio requirement, the mean absolute angular error of the method is less than $2^{\circ}$. It is further shown that this error is in part caused by other uncertainties such as the rotary joint phase properties, the rotary platform precision and the phase noise.

The actual requirements for the axial-ratio will depend on the specifics of the application. For a measurement of the time required for a single rotation (coarse measurement of angular velocity $\omega$ ), the phase error discussed is less important because the system only observes the time required to scribe the ellipse in the complex plane as described by (4). For a more precise continuous analysis of the rotary motion, the axial-ratio requirements will be stricter, but the literature offers numerous techniques to improve axial-ratios well below 1 dB, e.g. [12],[13].

\section{SENSOR FOR VARIABle DISTANCE - DuAL CP ANTENNAS}

A second important limitation of the sensor described in section III is the fact that it operates only for a fixed distance, i.e. a change of $\theta_{S Y S}$ caused by variable distance between the sensors causes a phase change which cannot be differentiated from the phase change due to rotation. At $\mathrm{GHz}$ frequencies, even a small change in range $r$ (see e.g. (2)) can cause a significant error. For instance at $3 \mathrm{GHz}$, a shift by $1 \mathrm{~mm}$ equals a phase change by $3.6^{\circ}$. For many industrial applications with small vibrations, this could be a significant limitation of the proposed method.

Therefore in this section, an enhancement that removes this limitation is introduced. It is shown that it is possible to achieve a linear relationship between the rotation angle and phase where the linear relationship is independent of the (variable) range between the antennas. This is achieved by introducing dual circularly-polarized antennas.

\section{A. Principle}

Let us consider a link between two dual-port antennas. Each antenna has one port for a right-hand (RH) circularpolarization and one port for left-hand (LH) circularpolarization. Between two such antennas, it is possible to establish independent RH and LH links. For the RH link Eq. (2) takes the form:

$$
H_{R}(f)=\frac{c \exp \left(-j 2 \pi \frac{f}{c} r\right)}{\mathrm{j} 2 \pi f r} H_{2 R X}(f) H_{1 R X}(f) \exp (j \varphi)
$$

For the LH Eq. (2) becomes

$$
H_{L}(f)=\frac{c \exp \left(-j 2 \pi \frac{f}{c} r\right)}{\mathrm{j} 2 \pi f r} H_{2 L X}(f) H_{1 L X}(f) \exp (-j \varphi)
$$

where $H_{i R X}$ and $H_{i L X}$ note that the transfer functions might differ between both antennas.

Division of (7) by (8) cancels out the distance term and yields:

$$
H_{R}(f) / H_{L}(f)=\frac{H_{2 R X}(f) H_{1 R X}(f)}{H_{2 L X}(f) H_{1 L X}(f)} \exp (j 2 \varphi)
$$

It is apparent that (9) does not contain any range term, i.e. the change of distance does not have any impact on the ratio $H_{R} / H_{L}$. However, it is noted that the phase term is doubled, i.e. $\Varangle\left(H_{R} / H_{L}\right) \propto 2 \varphi$. That means that a single physical rotation corresponds to two rotations in the complex plane. As a result, FM demodulation provides information about $2 \omega$.

\section{B. Proof-of-concept Measurement}

To prove the concept, two dual circularly-polarized antennas were prototyped according to [15]. Their shape is shown in Fig. 9 and they are designed to produce $\mathrm{RH}$ and $\mathrm{LH}$ circular-polarization at $2.5 \mathrm{GHz}$.

The antennas were positioned face-to-face at a distance $r$ apart, which varied from 30 to $45 \mathrm{~cm}$ in $5 \mathrm{~cm}$ steps. For each distance one antenna was rotated around $360^{\circ}$ in $5^{\circ}$ steps using a rotary platform. For each position, $H_{R}$ and $H_{L}$ were recorded. Fig. 10 shows the measured phase $\measuredangle\left(H_{R} / H_{L}\right)$ at $2.5 \mathrm{GHz}$ for

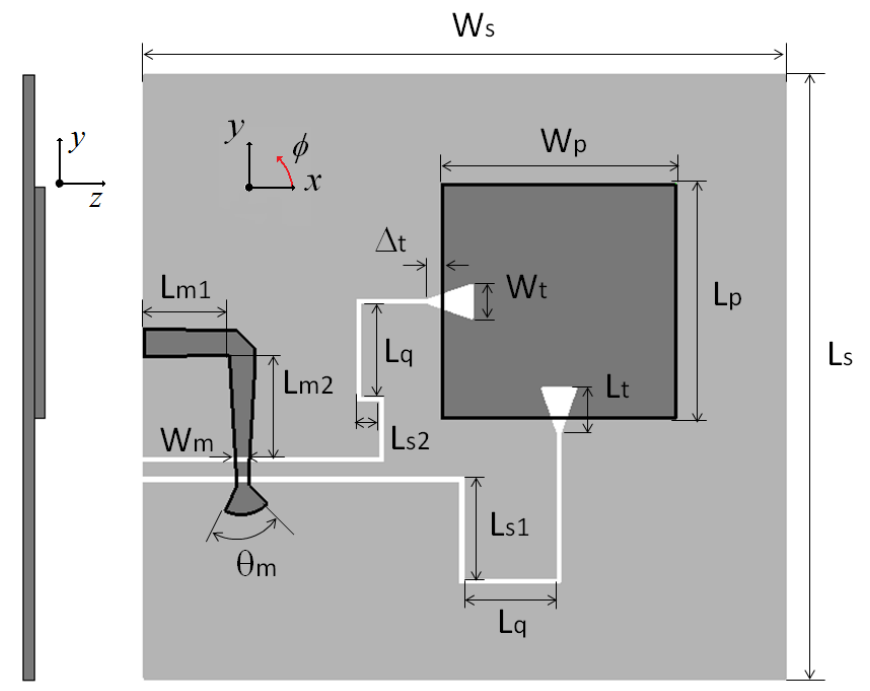

Fig. 9 Design of dual circularly-polarized antenna from [15]. Black line represent metalization edges on top layer, light grey color represents the ground plane, white color represents slots in ground plane. The dimensions as well as information about the substrate material can be found in [15]

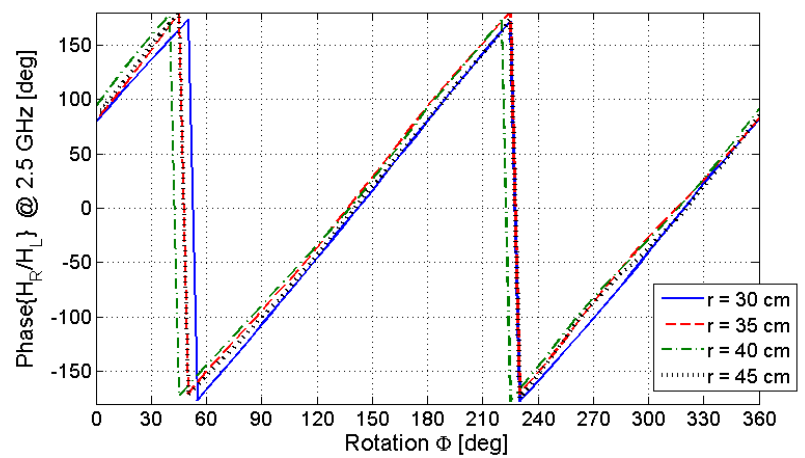

Fig. 10 Measured dependency of $H_{R} / H_{L}$ as a function of rotation angle. 
all 4 measurement distances $r$. This confirms the phase increments twice as fast as the physical rotation and that the phase $\measuredangle\left(H_{R} / H_{L}\right)$ is independent of the range. This is a significant result enabling the use of the proposed sensor concept for cases where the distance between the rotating object and the reader varies. The errors (differences among the lines) in Fig. 10 are caused by antenna imperfections, misalignment and platform positioning errors, however good agreement between the lines supports the theory from section IV.A.

\section{CONCLUSION}

This paper describes an alternative contactless method for measurement of angular velocity. It shows that the transfer function phase of a wireless link between two circularlypolarized antennas depends on the angle between the antennas. This is used to measure the angular velocity around the boresight axis between the antennas.

It is shown that the precision depends strongly on the axial ratio of both antennas and that a link with an $\mathrm{AR}<3 \mathrm{~dB}$ allows angular precision of less than $2^{\circ}$ and less than $1.5^{\circ}$ for $\mathrm{AR}<1 \mathrm{~dB}$. It is also believed that this is limited by imperfections of the measurement setup. Therefore, the actual precision of the method can be further improved.

The basic method works only for fixed distance links because a change in the distance also impacts the phase of the transfer function. This limitation can be removed by the use of antennas with both right- and left-hand circular polarization.

It is believed that this method will find applications in industrial settings. Firstly it enables continuous measurement of angular velocity with very high precision whilst using lowcost hardware.

Secondly, small changes to the hardware enable a wide range of measured angular velocities - from extremely slow to extremely high speeds (>100,000 rpm).

Thirdly, the wireless link can be re-used for other sensors to transmit their data whilst the angular velocity is being measured.

Lastly, a single receiver can support more than one transmitter, i.e. a single base station could allow simultaneous measurement of multiple rotating objects within its circularlypolarized beamwidth.

Future work will focus on extending the method towards passive tags so that neither a battery nor a signal source are required on the rotating object.

\section{ACKNOWLEDGMENT}

The work was funded by the Government of Ireland Fellowship in Engineering, Science and Technology funded by the Irish Research Council and by Science Foundation Ireland under Grants No. 10/CE/I1853 and No. 13/TIDA/I2746.

\section{REFERENCES}

[1] R. Bonert, "Design of a high performance digital tachometer with a microcontroller," Instrumentation and Measurement, IEEE Transactions on , vol.38, no.6, pp.1104,1108, Dec 1989
[2] S.J. Arif, M.S.J. Asghar, A. Sarwar, "Measurement of Speed and Calibration of Tachometers Using Rotating Magnetic Field," Instrumentation and Measurement, IEEE Transactions on , vol.63, no.4, pp.848,858, April 2014

[3] Xin Li, Junjie Qi, Qi Zhang, Yue Zhang, "Bias-tunable dual-mode ultraviolet photodetectors for photoelectric tachometer," Applied Physics Letters, vol.104, no.4, pp.041108-041108-4, Jan 2014

[4] J. Naqui, F. Martin, "Angular Displacement and Velocity Sensors Based on Electric-LC (ELC) Loaded Microstrip Lines," Sensors Journal, IEEE, vol.14, no.4, pp.939,940, April 2014

[5] J. Naqui, F. Martin, "Transmission Lines Loaded with Bisymmetric Resonators and Their Application to Angular Displacement and Velocity Sensors," IEEE Transactions on Microwave Theory and Techniques, vol. 61(12), pp. 4700-4713, Dec. 2013

[6] A. Ebrahimi, W. Withayachumnankul, S.F. Al-Sarawi, D. Abbott, D., "Metamaterial-Inspired Rotation Sensor With Wide Dynamic Range," Sensors Journal, IEEE, vol.14, no.8, pp.2609,2614, Aug. 2014

[7] V. Sipal, A. Narbudowicz, M. J. Ammann, "Using near-field coupled circularly polarised antennas as frequency-independent variable phase shifters," Electronics Letters, vol.50, no.11, pp.788-790, May. 2014

[8] Y. Duroc, Vuong Tan-Phu, S. Tedjini, "A Time/Frequency Model of Ultrawideband Antennas," Antennas and Propagation, IEEE Transactions on, vol.55, no.8, pp.2342-2350, Aug. 2007

[9] S. Gao, Qi Luo, Fuguo Zhu, Circularly polarized antennas, Wiley-IEEE Press, January 2014

[10] H.D. Foltz, J.S. McLean, A. Medina, A., J.H. Alvarez Jerkov, "Lack of Rotation Invariance in Short-Pulse Communication Between Broadband Circular-Polarization Antennas," Antennas and Propagation, IEEE Trans., vol.58, no.11, pp.3747-3751, Nov. 2010

[11] Kline, P.A.: "Atomic Clock Augmentation For Receivers Using the Global Positioning System," PhD. Thesis, Virginia Tech, 1997

[12] Nasimuddin, X. Qing, Z.N. Chen, "Compact Circularly Polarized Symmetric-Slit Microstrip Antennas," Antennas and Propagation Magazine, IEEE, Volume: 53 , Issue: 42011 , Page(s): 63 - 75

[13] Li Rong-Lin Li, V.F. Fusco, "Printed figure-of-eight wire antenna for circular polarization," Antennas and Propagation, IEEE Transactions on, Volume: 50 , Issue: 102002 , Page(s): 1487 - 1490

[14] V. Sipal, B. Allen, D. Edwards, B. Honary, "Twenty years of ultrawideband: Opportunities and challenges," Communications, IET, vol.6, no.10, pp.1147,1162, July 32012

[15] A. Narbudowicz, Xiulong Bao, M.J. Ammann, "Dual CircularlyPolarized Patch Antenna Using Even and Odd Feed-Line Modes," Antennas and Propagation, IEEE Transactions on, vol.61, no.9, pp.4828,4831, Sept. 2013 